\title{
Metodología para aprender programación funcional en ingeniería de sistemas aplicando teoría de aprendizaje por descubrimiento
}

\author{
Omar Iván Trejos-Buriticá \\ Facultad de Ingenierías, Universidad Tecnológica de Pereira,Pereira, Colombia.omartrejos@utp.edu.co
}

\begin{abstract}
Resumen-- En el presente artículo se examinan las posibles ventajas que se incorporan en un curso de programación de computadores de Ingeniería de Sistemas de primer semestre cuando se implementa una metodología basada en la teoría por descubrimiento y se sirve durante las sesiones prácticas. La metodología se enmarcó en la investigación educativa de carácter cualitativa, articulada con un estudio de casos instrumental y múltiple. Los datos se obtuvieron en las sesiones prácticas durante las cuales se recolectó tanto la opinión de los estudiantes como la observación de sus avances individuales en el desarrollo y solución de los ejercicios propuestos. Los resultados evidencian aprendizajes más significativos cuando se permite que el estudiante, partiendo de los conocimientos previos, pueda inferir el nuevo conocimiento de la mano de explicaciones magistrales que les brinden las semillas necesarias para poder avanzar de manera autónoma. De la misma manera, esta metodología posibilita independencia académica y motivación a los estudiantes para otros aprendizajes en su proceso de formación como ingenieros.
\end{abstract}

Palabras clave-- Aprendizaje por descubrimiento; ingeniería de sistemas; metodología; paradigma de programación; programación de computadores; programación funcional

Recibido: 6 de septiembre de 2015. Revisado: 1 de noviembre de 2015. Aceptado: 25 de noviembre de 2015

\section{Methodology to learn functional programming in systems engineering using discovery learning theory}

Abstract-- In this article we examine possible advantages to apply Discovery Learning in the first programming computer course in Systems Engineering during the practical sessions. The methodology was framed in educational research with qualitative character based in multiple cases study. The data were collected during the practical sessions from the opinion of the students and the observation of their advances in the resolution of computational problems. The results show more significant and meaningful learning taking the previous knowledge and with the possibility to inferring new knowledge. In the same way, this methodology brings academic independence and motivation for students to access another learnings in the courses of their studies.

Keywords-- Learning for discovering, systems engineering, methodology, programming paradigm, computer programming, functional programming

\section{Introducción}

El conocimiento, apropiación, asimilación y aplicación de las teorías de aprendizaje han sido una de las preocupaciones que se tienen en los programas de Ingeniería [1] toda vez que un docente ingeniero está capacitado, por su formación disciplinar, para ser ingeniero y pocas veces, por ese mismo camino, está capacitado para ser docente. De allí que ha de acceder a conocimientos teóricos que si bien no son de su competencia profesional si lo son de su necesidad como docente de ingeniería [2]. Del conocimiento de estas teorías se desprenden las estrategias de enseñanza para que los estudiantes retengan y apliquen los conocimientos que se les quiere servir.

Normalmente el profesor acude a las metodologías tradicionales como son la exposición magistral para intentar llevar al cerebro de los estudiantes un conocimiento de orden tecnológico. Sin embargo, cuando se trata de cursos de programación de computadores, en los cuales el uso de tecnologías computacionales es imprescindible, la necesidad de dinamizar la exposición magistral lleva a que el docente ingeniero deba acudir a teorías de aprendizaje para poder alcanzar los logros y objetivos establecidos en un curso de programación de computadores [3] esperando que le retención y aplicación de los conocimientos se pueda ubicar en la memoria a largo plazo de manera que sirva de soporte para los posteriores usos que la formación disciplinar exija. Una de ellas es la teoría del aprendizaje por descubrimiento [4] íntimamente atada a la teoría de aprendizaje significativo [5].

La aplicación de las mejores estrategias que se extraigan de estas teorías constituye el día a día de los docentes ingenieros en la búsqueda de poder llegar con su conocimiento al estudiante de forma que éste lo asimile, lo apropie, lo aplique y lo evalúe. Esto lleva a que se justifique en un programa de ingeniería la búsqueda de todas las estrategias posibles acudiendo a las teorías de aprendizaje de que se dispongan para buscar caminos que propendan por un aprendizaje sólido tal que el estudiante, al resolver un problema, tenga la suficiente conciencia de la lógica que los sustenta [7]. Algunas veces demuestran poca habilidad para aprender de manera autónoma y menos habilidad para descubrir el conocimiento que subyace a una nueva temática que debe abordarse como parte de la formación disciplinar como ingeniero [7]. Proponer caminos para subsanar estas dificultades es el propósito de este artículo y de su investigación asociada.

De alguna forma, es tradición que el estudiante aprenda de forma memorística los nuevos conocimientos que debe adquirir. El problema se presenta cuando este conocimiento recientemente adquirido se ubica en la memoria a corto plazo y se convierte en un conocimiento tan volátil y tan frágil que 


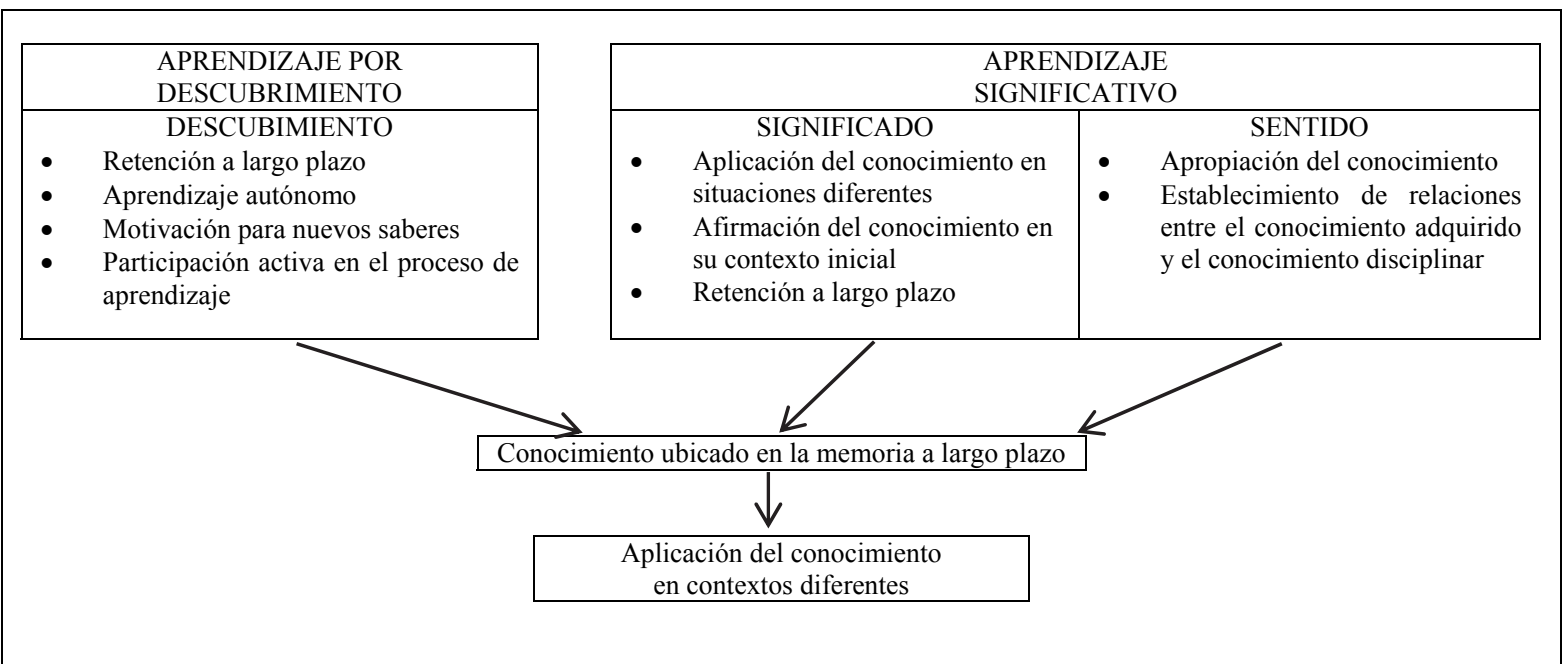

Gráfica 1. Relación Descubrimiento - Significado - Sentido

Fuente: Elaboración propia

prontamente se olvida [3]. Es allí en donde la teoría del aprendizaje por descubrimiento aporta elementos de juicio que posibilitan que, precisamente por la vía del descubrimiento realizado por el mismo estudiante, éste se pueda ubicar en la memoria a largo plazo confiriéndole significado y sentido de manera que no solo el estudiante comprenda y asimile la situación en donde lo aplicó sino que, además, pueda encontrar nuevas situaciones en donde este conocimiento llegue a ser solución de diferentes situaciones tal como lo sugiere la formación por competencias y como se muestra en la Gráfica 1.

Otro aporte que posibilita la teoría del aprendizaje por descubrimiento es la facilidad de abrir caminos para el aprendizaje autónomo y para que el mismo estudiante, de manera voluntaria, tome parte activa de su proceso de aprendizaje como lo indican las estrategias active learning. En este contexto existe la posibilidad de que el aprendizaje por descubrimiento proporcione en gran medida elementos de juicio requeridos para que el aprendizaje de la programación funcional encuentre un camino más expedito entre el acompañamiento del docente y el conocimiento adquirido por el estudiante.

La pregunta de investigación que se plantea es la siguiente: ¿es posible encontrar estrategias que posibiliten mejorar el aprendizaje de la programación funcional a partir de la aplicación de la teoría del aprendizaje por descubrimiento en un programa de ingeniería de sistemas siendo este paradigma el corpus que constituye el primer curso de programación? La respuesta a esta investigación es el contenido del presente artículo en el cual se exploran esos caminos, se ponen en la escena académica y se evalúan a partir de diferentes pruebas realizadas con los estudiantes en las cuales se acude no sólo a su valoración cuantitativa sino también a su opinión cualitativa.

En cuanto a la posición epistemológica de la investigación planteada puede decirse que se acude a la necesidad que tiene el ser humano de DESCUBRIR [8] como un posible mecanismo para afianzar los conocimientos en la memoria a largo plazo y poder llegar a aplicarlos con suficiencia en situaciones similares o diferentes a aquellas en las cuales se contextualizó inicialmente dicho conocimiento sin desconocer que cada estudiante tiene un conjunto de vivencias y situaciones que, desde los tres contextos escolares, le permiten crear un marco de referencia que puede ser diferente a los demás para asimilar conocimientos disciplinares. Además no ha de desconocerse que la organización de cada cerebro es diferente incluso así tenga las mismas vivencias [9].

Este artículo es uno de los productos asociados al proyecto de investigación "Desarrollo de un modelo metodológico para el aprendizaje de la programación imperativa en Ingeniería de Sistemas basado en aprendizaje significativo, aprendizaje por descubrimiento y el modelo $4 \mathrm{Q}$ de preferencias de pensamiento" aprobado por la Vicerrectoría de Investigaciones, Innovación y Extensión de la Universidad Tecnológica de Pereira bajo el código 6-16-13.

\section{Teoría}

Como metodología se puede definir el camino o, también, el conjunto de procesos y procedimientos que, siendo altamente racionales, son usados para lograr un determinado objetivo y un conjunto de objetivos y que propende por fortalecer las leyes o conclusiones hasta las cuales llegue la investigación científica, los conocimientos que requieran determinadas habilidades y determinados cuidados [10]. La palabra metodología hace referencia a un término demasiado amplio dado que acude a un concepto que, mayormente, puede ser reemplazado por la palabra método.

Etimológicamente la palabra metodología significa "el estudio del camino que lleva más allá" y es por esto que esta palabra acude a un concepto propio de la investigación científica que busca cumplir los objetivos que se han planteado en la ciencia. Todo esto lleva a pensar que la metodología, como concepto, puede aproximarse a un conjunto de procedimientos que establece el conocimiento que se deriva de una investigación científica o que determina un específico rumbo doctrinal. 
Por su parte, la programación funcional se puede definir como un paradigma de la programación declarativa que se basa en la definición y uso de las funciones matemáticas en contraposición (no necesariamente antagónica) a la programación imperativa. En la programación funcional priman las funciones como núcleos básicos de trabajo que posibilitan obtener resultados a partir de determinados procesos, operaciones y estructuras [11]. En la programación imperativa su esencia está fundamentada en el concepto de estados asociado a la utilización de variables que son las que almacenan la información y cuya interacción se logra a partir de la buena utilización de instrucciones de lenguajes de programación que buscan alterar los contenidos de las variables, interactuar con ellos y operarlos de forma que, en un momento dado, se obtenga el resultado que se espera [12].

Es de anotarse que la utilización de variables ( $\mathrm{y}$ de su concepto de "estado" asociado) implica efectos colaterales en el desarrollo y aplicación de un algoritmo en términos de lenguajes de programación [13]. En el caso de la programación funcional, ésas se alimentan de argumentos que son los que nutren una función para que esta funcione -valga esta redundancia. La eliminación de los efectos colaterales secundarios que involucra la programación imperativa permite entender y, en determinados casos, llegar a predecir el comportamiento de un programa específico.

Los lenguajes funcionales han tenido un gran uso en lo académico ya que permiten fortalecer el concepto de función y sus características, fomentar la notación formal y establecer un nexo íntimo entre las matemáticas y la programación de computadores [14]. Algunos de los lenguajes funcionales (o que soportan programas puramente funcionales) son Scheme, Caml, Scala y Haskell. La programación funcional también ha tenido grandes aplicaciones en campos como la estadística, las matemáticas y el análisis financiero. Es de anotar que la filosofía de la programación funcional (estructuración de una solución algorítmica a través de funciones, construcción de funciones sin incluir el concepto de "estados", aprovechamiento de las características de las funciones llamados, recursividad, operatividad) puede implementarse también en lenguajes de programación imperativos e, incluso, a partir del concepto de métodos en la Programación Orientada a Objetos llegan a ser de gran utilidad en este otro paradigma [15].

En referencia con la teoría de aprendizaje por descubrimiento se puede decir que fue desarrollada por sicólogo norteamericano Jerome Seymour Bruner bajo la óptica constructivista también conocida como aprendizaje heurístico. En esta teoría se busca promover en el alumno una buena capacidad de adquirir los conocimientos por sí solo. La forma como se empezó a concebir la educación, desde esta teoría, representó un cambio significativo en la aplicación de métodos de enseñanza tradicionales pues los contenidos se comenzaron a mostrar de forma parcial y no de manera final y definitiva, como era la costumbre. La teoría sugiere que él debe DESCUBRIR progresivamente lo que falta o lo que sigue tomando como punto de partida lo que proponga el profesor y a partir de sus descubrimientos, el conocimiento quedará enraizado en la memoria a largo plazo con significado y sentido.

Según Bruner, los estudiantes aprenden más si el proceso de aprendizaje es producto de un descubrimiento guiado que implique exploración promovida por la curiosidad y la fascinación, dos partes consideradas de gran importancia para generar motivación y así poder aproximarse al aprendizaje autónomo. Bruner también plantea que el profesor no tiene como función, en el aula, la presentación de contenidos desde el principio hasta el final sino que debería aplicar estrategias que posibiliten estimular a sus estudiantes en relación con la observación, comparación, el desarrollo del análisis, el hallazgo de semejanzas y diferencias y otros elementos que son los que, a la postre, permitirán descubrir.

El aprendizaje por descubrimiento posibilita la superación de las limitantes que implican las estrategias de aprendizaje tradicional (memorístico o mecanicista), facilita la promoción en los alumnos para pensar por sí solos formulando hipótesis e intentando verificarlas a través de métodos establecidos y aumenta la autoestima y sensación de seguridad en los estudiantes a partir del hallazgo y planteamiento de soluciones creativas en relación con problemas planteados.

Se le critica a la teoría de aprendizaje por descubrimiento la falsa visión sobre los beneficios de esta teoría dado que, según Ausubel no necesariamente cuando se aprende por descubrimiento se involucra significado ni tampoco es totalmente cierto que aprender memorísticamente sea necesariamente mecánico. Todo dependerá de la forma como la información es captada por los sentidos y de cómo los procesa el cerebro así como los almacene.

\section{Metodología y Resultados}

La metodología utilizada en el desarrollo de esta investigación se explica en la Tabla 1 en donde se explican las fases planteadas con su correspondiente descripción.

Tabla 1.

Descripción de fases de la metodología

\begin{tabular}{|c|c|}
\hline Fase & Descripción \\
\hline 1 & $\begin{array}{l}\text { Lo primero que se hizo fue explicar a los estudiantes el proceso } \\
\text { que se iba a realizar, sus objetivos y sus características y la manera } \\
\text { como ellos iban a participar de manera activa }\end{array}$ \\
\hline 2 & $\begin{array}{l}\text { Se diseñaron instrumentos que permitieran caracterizar a los } \\
\text { estudiantes en tres niveles: muy talentosos, mediano talentosos y } \\
\text { bajo talentosos, en relación con la programación de computadores }\end{array}$ \\
\hline 3 & $\begin{array}{l}\text { Se dividió el grupo de estudiantes en dos subgrupos de a } 9 \\
\text { estudiantes. En ambos grupos quedaron un número equivalente de } \\
\text { estudiantes de acuerdo con la caracterización de la fase } 2 \text {. Un } \\
\text { grupo se atenderá con metodología tradicional (1) y el otro grupo } \\
\text { con metodología basada en aprendizaje por descubrimiento (2) }\end{array}$ \\
\hline 4 & $\begin{array}{l}\text { Se establecieron horarios paralelos en ambos grupos de forma que } \\
\text { se pudieran explicar en tres sesiones los tres temas a abordar } \\
\text { estando el docente con un grupo en la } 1^{\mathrm{a}} \text { hora y con el otro grupo } \\
\text { en la } 2^{\mathrm{a}} \text { hora. }\end{array}$ \\
\hline 5 & $\begin{array}{l}\text { Finalizadas las tres sesiones, se realizó la misma evaluación acerca } \\
\text { de los temas vistos en ambos grupos }\end{array}$ \\
\hline 6 & $\begin{array}{l}\text { Se consultó la opinión de cada uno de los estudiantes en relación } \\
\text { con la metodología utilizada en cada grupo }\end{array}$ \\
\hline 7 & $\begin{array}{l}\text { Se entregaron los resultados de las evaluaciones y se socializó la } \\
\text { experiencia con los estudiantes }\end{array}$ \\
\hline
\end{tabular}

Fuente: Elaboración propia 
Tabla 2.

Temas y metodologías abordadas

\begin{tabular}{|c|c|c|c|}
\hline Tema & $\begin{array}{l}\text { Nombre } \\
\text { General }\end{array}$ & $\begin{array}{c}\text { Tema } \\
\text { Explicado }\end{array}$ & $\begin{array}{l}\text { Tema por } \\
\text { Descubrir }\end{array}$ \\
\hline 1 & $\begin{array}{l}\text { Resolución } \\
\text { de } \\
\text { problemas }\end{array}$ & $\begin{array}{l}\text { Se plantea una metodología para que, a } \\
\text { partir de la formulación de un problema, } \\
\text { extender sus fronteras de efectos } \\
\text { negativos }\end{array}$ & $\begin{array}{l}\text { Cómo resolver sistemáticamente un problema } \\
\text { de acuerdo a unos pasos establecidos }\end{array}$ \\
\hline 2 & $\begin{array}{l}\text { Notación } \\
\text { Computacional }\end{array}$ & $\begin{array}{l}\text { Se presenta la notación en posfijo } \\
\text { (operandos - operador) y se hacen } \\
\text { conversiones y operaciones con las } \\
\text { expresiones finales }\end{array}$ & $\begin{array}{l}\text { Notación polaca o notación en prefijo que es la } \\
\text { que se utiliza en la programación funcional }\end{array}$ \\
\hline
\end{tabular}

Fuente: Elaboración propia

Tabla 3.

Evaluación realizada

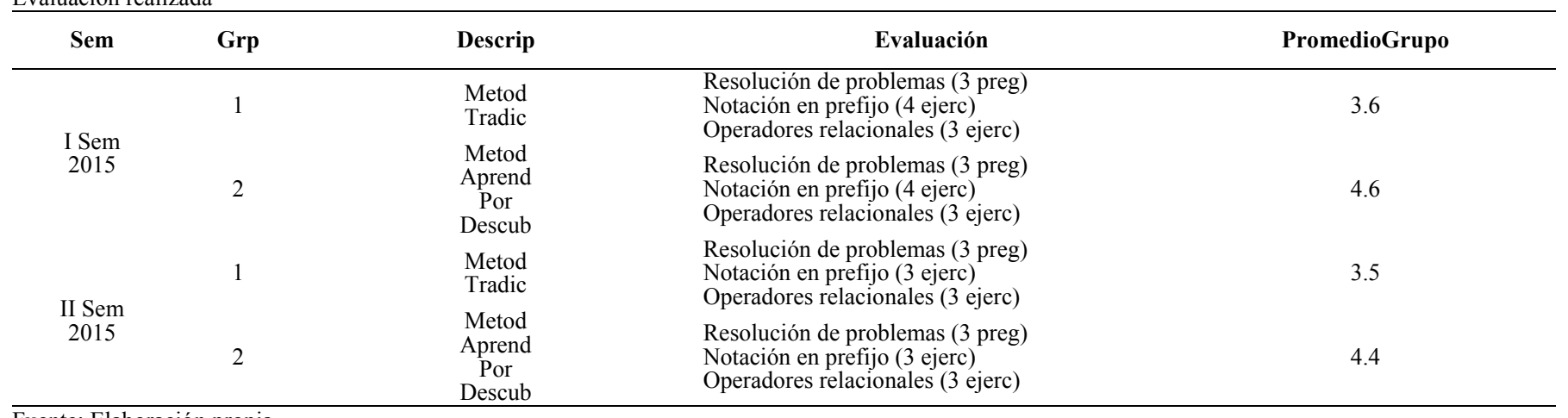

Fuente: Elaboración propia

Tabla 4.

$\underline{\text { Retroalimentación por parte de los estudiantes }}$

\begin{tabular}{|c|c|c|c|c|}
\hline \multirow[b]{2}{*}{ Grp } & \multirow[b]{2}{*}{ Descrip } & \multicolumn{3}{|c|}{ Opiniones de los estudiantes } \\
\hline & & $\begin{array}{c}\text { Tema } 1 \\
\text { Resolución de Problemas }\end{array}$ & $\begin{array}{c}\text { Tema } 2 \\
\text { Notación Computacional }\end{array}$ & $\begin{array}{c}\text { Tema } 3 \\
\text { Operadores }\end{array}$ \\
\hline 1 & $\begin{array}{l}\text { Metod } \\
\text { Tradic }\end{array}$ & $\begin{array}{ll} & \text { Lo normal } \\
- & \text { Me pareció muy obvio } \\
- & \text { Lo que está en los libros } \\
- & \text { Como siempre } \\
- & \text { Más o menos } \\
- & \text { Entendible }\end{array}$ & $\begin{array}{ll}- & \text { Un poquito enredado } \\
\bullet & \text { Podría ser más sencillo } \\
\bullet \quad & \text { Se requieren muchos ejercicios } \\
& \text { Hay quacticar }\end{array}$ & $\begin{array}{ll}\text { - } & \text { Fácil de entender } \\
\text { - } & \text { La explicación fue muy buena } \\
\text { - } & \text { ¿Podríácil el tema } \\
& \text { Per más fácil? }\end{array}$ \\
\hline 2 & $\begin{array}{l}\text { Metod } \\
\text { Aprend } \\
\text { X } \\
\text { Descub }\end{array}$ & $\begin{array}{l}\text { - } \quad \text { Fue muy fácil concluir el método } \\
\text { de Polya } \\
\text { - } \quad \text { Me pareció muy interesante } \\
\text { - } \quad \text { Todo lo descubrí } \\
\text { - } \quad \begin{array}{l}\text { Practicamos bastante } \\
\text { entendiendo }\end{array} \\
\end{array}$ & $\begin{array}{ll}\text { - } & \text { Muy fácil el tema } \\
\text { Cambiar la posición del } \\
\text { operador fue muy sencillo } \\
\text { - } \quad \text { Así cualquiera se hace } \\
\text { ingeniero de sistemas } \\
\text { - } \quad \text { La explicación fue maravillosa } \\
\text { - Sencillito, como dice el profe }\end{array}$ & $\begin{array}{ll}\text { - } & \text { Muy sencillo utilizar } \\
\text { - } & \text { Meradores } \\
\text { Muy fácil combinar notación } \\
\text { prefijo y operadores } \\
\text { - } \quad \text { El tema es demasiado fácil } \\
\text { demasiado fácil }\end{array}$ \\
\hline
\end{tabular}

Fuente: Elaboración propia

Los temas que se abordaron y la metodología utilizada se describen en la Tabla 2 de acuerdo con lo planteado en los objetivos de la presente investigación.

Se realizó una misma evaluación escrita en ambos grupos. La descripción de la evaluación escrita realizada y el promedio de cada grupo se presentan en la Tabla 3 de acuerdo a lo visto en las clases magistrales.

La opinión de los estudiantes en relación con los temas se recogió a partir de una pregunta abierta realizada por escrito: Exprese libremente su opinión acerca de la metodología utilizada. Algunas apartes de estas opiniones se muestran en la Tabla 4.

\section{Discusión}

En referencia con la metodología descrita en la Tabla 1, se explican en la Tabla 5 los objetivos o propósitos que se persiguieron con cada una de las fases implementadas para el desarrollo de la investigación. 
Tabla 5 .

Objetivos de cada fase

\begin{tabular}{|c|c|c|}
\hline Fase & Descripción & Objetivos \\
\hline 1 & $\begin{array}{l}\text { Lo primero que se hizo fue } \\
\text { explicar a los estudiantes el } \\
\text { proceso que se iba a realizar, sus } \\
\text { objetivos y sus características y } \\
\text { la manera como ellos iban a } \\
\text { participar de manera activa }\end{array}$ & $\begin{array}{l}\text { Involucrar conscientemente a } \\
\text { los estudiantes en el proceso de } \\
\text { forma que pudieran conocer su } \\
\text { avance y, así mismo, pudieran } \\
\text { capitalizar cada uno de los } \\
\text { pasos que se dieran }\end{array}$ \\
\hline 2 & $\begin{array}{l}\text { Se diseñaron instrumentos que } \\
\text { permitieran caracterizar a los } \\
\text { estudiantes en tres niveles: muy } \\
\text { talentosos, mediano talentosos y } \\
\text { bajo talentosos, en relación con } \\
\text { la programación de } \\
\text { computadores }\end{array}$ & $\begin{array}{l}\text { Tener un panorama más claro } \\
\text { en relación con el rendimiento } \\
\text { académico de cada uno de los } \\
\text { estudiantes de forma que la } \\
\text { segmentación pudiera hacerse } \\
\text { con unas bases más objetivas }\end{array}$ \\
\hline 3 & $\begin{array}{l}\text { Se dividió el grupo de } \\
\text { estudiantes en dos subgrupos de } \\
\text { a } 9 \text { estudiantes. En ambos } \\
\text { grupos quedaron un número } \\
\text { equivalente de estudiantes de } \\
\text { acuerdo con la caracterización } \\
\text { de la fase } 2 \text {. Un grupo se } \\
\text { atenderá con metodología } \\
\text { tradicional (1) y el otro grupo } \\
\text { con metodología basada en } \\
\text { aprendizaje por descubrimiento } \\
\text { (2) }\end{array}$ & $\begin{array}{l}\text { Balancear la distribución de } \\
\text { estudiantes de acuerdo a su } \\
\text { rendimiento académico y } \\
\text { establecer un paralelo que } \\
\text { permitiera comparar resultados } \\
\text { entre los do grupos } \\
\text { seleccionados: uno con la } \\
\text { metodología tradicional y el } \\
\text { otro con la metodología de } \\
\text { aprendizaje por descubrimiento }\end{array}$ \\
\hline 4 & $\begin{array}{l}\text { Se establecieron horarios } \\
\text { paralelos en ambos grupos de } \\
\text { forma que se pudieran explicar } \\
\text { en tres sesiones los tres temas a } \\
\text { abordar estando el docente con } \\
\text { un grupo en la } 1^{\text {a }} \text { hora y con el } \\
\text { otro grupo en la } 2^{\mathrm{a}} \text { hora. }\end{array}$ & $\begin{array}{l}\text { Propiciar las mismas } \\
\text { condiciones (de forma, tiempo } \\
\text { y lugar) para que ambos } \\
\text { subgrupos recibieran las clases } \\
\text { sin que existieran factores que } \\
\text { enturbiaran la investigación }\end{array}$ \\
\hline 5 & $\begin{array}{l}\text { Finalizadas las tres sesiones, se } \\
\text { realizó la misma evaluación } \\
\text { acerca de los temas vistos en } \\
\text { ambos grupos }\end{array}$ & $\begin{array}{l}\text { Establecer parámetros que } \\
\text { permitan verificar, por escrito, } \\
\text { lo comprendido y aprendido de } \\
\text { los temas vistos }\end{array}$ \\
\hline 6 & $\begin{array}{l}\text { Se consultó la opinión de cada } \\
\text { uno de los estudiantes en } \\
\text { relación con la metodología } \\
\text { utilizada en cada grupo }\end{array}$ & $\begin{array}{l}\text { Retroalimentar el proceso con } \\
\text { la opinión de los protagonistas } \\
\text { del mismo que son los propios } \\
\text { estudiantes }\end{array}$ \\
\hline 7 & $\begin{array}{l}\text { Se entregaron los resultados de } \\
\text { las evaluaciones y se socializó la } \\
\text { experiencia con los estudiantes }\end{array}$ & $\begin{array}{l}\text { Compartir la experiencia con } \\
\text { los mismos alumnos y escuchar } \\
\text { al respecto los aportes, críticas } \\
\text { y observaciones que quieran } \\
\text { hacer }\end{array}$ \\
\hline
\end{tabular}

Fuente: Elaboración propia

Desde la $1^{\text {a }}$ fase hasta la última se buscaba con esta metodología, además de encontrar un camino más llano para alcanzar los objetivos de aprendizaje, permitir que el estudiante conociera y aprovechara cada avance y, de esa forma, se involucrara activamente en su desarrollo.

En referencia con los temas abordados, que se presentan en la Tabla 2, vale la pena anotar que la selección de dichos temas (resolución de problemas, notación computacional y operadores) obedece a algunas dificultades detectadas por el autor de este artículo a lo largo de sus más de veinte años de experiencia docente en el área de la programación de computadores. La resolución de problemas es un tema fácilmente digerible y entendible, sin embargo, su aplicación metodológica no lo es tanto dado que el estudiante normalmente conoce las metodologías para resolución de problemas pero al momento de aplicarlas acude directamente a la construcción de programas a través del código.
Por su parte la notación computacional, y exactamente la transformación de la tradicional notación infijo (que es la que conocemos desde primaria en la cual el operador se ubica entre los operandos) a la notación en prefijo (en la cual el operador se ubica antes de los operandos) involucra ciertas particularidades que, de una u otra forma, representan dificultades para el estudiante. No es despreciable el hecho de que el estudiante, en el $1^{\circ}$ semestre de una carrera profesional, viene de interactuar por más de 11 años con la notación infijo y se le cambia la estructura (por notación prefijo) tan solo en unos meses. Esa comprensión involucra complejidades que vale la pena tener en cuenta desde todo punto de vista en un curso inicial de programación funcional.

Por último la utilización de los operadores, tercer tema seleccionado para aplicar metodología de aprendizaje por descubrimiento, se ha incluido en esta selección debido a que si bien la comprensión de los operadores relacionales es casi natural dada la experiencia que el estudiante trae de sus ciclos formativos en primaria y secundaria, no es así tan simple cuando se trata de utilizar operadores lógicos pues estos tienen una dinámica más interesante $\mathrm{y}$, para el estudiante, más compleja a pesar de ser más sencillos y de aproximarse más a la lógica natural (lógica humana).

La Tabla 2 adicionalmente presenta la estrategia utilizada para propiciar el camino para que el estudiante, basado en sus conocimientos previos, "descubra" lo que realmente se le quiere servir como parte del contenido temático de la asignatura. Por el lado de la resolución de problemas, se le explica al estudiante una metodología hipotética destinada a complicar el escenario en el cual tiene efecto un determinado problema. En la aplicación de la metodología, es el estudiante quien debe encontrar caminos lógicos que le permitan plantear una forma de resolver el problema, es decir, avanzar en el sentido contrario al visto en clase.

En lo que corresponde al "descubrimiento" de la notación computacional se le sirve al estudiante las características, conversión, resolución e interacción con la notación en posfijo (aquella en la cual el operador se ubica después de los operandos) y se le indica al estudiante que, por su propia cuenta, plantee lo que se requiere -en conversión, resolución e interacción- para hacer efectiva la notación en prefijo (o sea aquella en la cual el operador se ubica antes de los operandos). Si bien pareciera que solo es cambiar de posición un operador, la realidad es que no es tan simple, pues el proceso de conversión de una expresión, el recorrido sobre el árbol binario, la obtención y verificación de la nueva expresión y su mecánica de resolución son completamente distintos. Incluso el mismo estudiante (en un porcentaje bastante alto) descubre la manera como la notación posfija puede ser tratada como prefija -o viceversa- $y$ de esa forma encuentra un punto en donde ambas notaciones se encuentran.

Los operadores relacionales y booleanos se estudian a partir de los operadores aritméticos en su ubicación, notación, descripción, uso, simplificación, distribución y obtención de resultados. Si los operadores aritméticos originan resultados numéricos, los operadores relacionales y booleanos originan resultados lógicos y ese simple detalle imprime una diferencia significativa en la comprensión del uso de estos operadores 
debido a que los resultados aritméticos se pueden operar aritméticamente para obtener otros resultados mientras que los resultados lógicos se pueden operar pero bajo reglas lógicas que son un poco diferentes.

De esta forma se buscó que a través de la "complicación" de los problemas, los estudiantes pudieran descubrir el camino para resolverlos; a través de la notación posfijo descubrieran la notación prefijo y a través de los operadores aritméticos descubrieran los operadores relacionales y lógicos operando bajo la notación en prefijo.

La Tabla 3 muestra los resultados cuantitativos que se derivan de la evaluación realizada a cada uno de los subgrupos en el respectivo semestre. En ella se puede observar que el promedio de la evaluación es superior en el subgrupo con el cual se trabajó bajo metodología de aprendizaje por descubrimiento en comparación con el subgrupo con el cual se trabajó la metodología tradicional. La diferencia en el promedio es notoria si se tiene en cuenta que es de 1 unidad en el I semestre de 2015 y de casi 1 unidad (0.9) en el II semestre de 2015. Las inferencias que se pueden derivar de los resultados de la Tabla 3 se revalidan con las opiniones cualitativas de la Tabla 4 pues en ella las opiniones de los estudiantes bajo metodología tradicional destacan, por momentos, cualidades del docente lo cual hace que el proceso de aprendizaje dependa de la capacidad del profesor mientras que los estudiantes bajo metodología de aprendizaje por descubrimiento destacan la metodología como tal lo cual permite que, al margen de las cualidades del docente es posible extrapolar esta metodología con sus estrategias y actividades para que se aplique en otras asignaturas y es allí en donde se establece la gran diferencia.

\section{Conclusiones}

En este tipo de investigaciones todo parece indicar que, cuando se involucra a los estudiantes enterándolos de cada una de las fases que se van a cubrir y de los logros que se quieren alcanzar, es mucho más fácil lograr objetivos de aprendizaje sea que estén mediados por una teoría como la del aprendizaje por descubrimiento que ha servido de base para esta investigación o cualquier otra teoría de aprendizaje. Tener un mecanismo objetivo que permita caracterizar a los estudiantes para poder realizar segmentaciones de grupos balanceadas forma parte de los criterios e instrumentos con que se debe contar para aproximarse a resultados mucho más confiables hablando en términos de investigación educativa tecnológica.

El desarrollo de estos procesos investigativos con grupos paralelos implica intentar mantener al máximo las condiciones externas de todo tipo de forma que los subgrupos que se quieran estudiar tengan los mismos factores externos tal que nada afecte el desarrollo de la investigación. Cualquier pequeño cambio aparente puede ser significativamente influyente en el desarrollo de este tipo de proyectos de investigación y por lo tanto puede llegar a influir sesgando los resultados. La evaluación escrita o bajo cualquier otro tipo de modalidad siempre será una necesidad para validar lo aprendido y para verificar si efectivamente este tipo de procesos han tenido el impacto esperado. El diseño de la evaluación ha de ser lo más objetiva posible de forma que se pueda confiar en los resultados obtenidos toda vez que la investigación esté en rima con la temática, las estrategias, las dinámicas y los conceptos involucrados.

Todo parece indicar que así como es importante enterar a los estudiantes de las fases, objetivos y propósitos de este tipo de investigaciones con el ánimo de que ellos participen activamente, también lo es presentarles los resultados obtenidos, los logros alcanzados y abrir espacios para que ellos opinen, critiquen, retroalimenten y enriquezcan el proceso como tal, al fin y al cabo ellos son los protagonistas y razón de ser de estas investigaciones. Finalmente es de anotar que este tipo de experiencias investigativas vale la pena confrontarlas por lo menos en dos semestres consecutivos para validar los resultados y poder aumentar la confiabilidad de los mismos en los términos en los que la investigación científica establece. Tampoco se puede desconocer que la teoría del aprendizaje por descubrimiento posibilita caminos de aproximación al conocimiento bastante sencillos para los estudiantes en su etapa de formación profesional en un programa de ingeniería.

\section{Bibliografía}

[1] Vega, A.M. y Espinel, A., Aspectos fundamentales para la enseñanza de la programación básica en ingeniería. Revista Avances en Sistemas e Informática, 7, pp. 7-13. 2010.

[2] Fincher. S., ¿What are we doing when we teach programming? 29th ASEE/IEEE Frontiers in Education Conference. San Juan, Puerto Rico. 10-13 de Noviembre de 1999, Sesión 12ª4.

[3] Trejos, O.I., Significado y competencias. Pereira. Papiro, 2013.

[4] Bruner, J., Actos de significado: Mas allá de la revolución cognitiva. Madrid. Alianza Editorial, 2009

[5] Ausubel, D., Sicología educativa: Un punto de vista cognoscitivo. Ciudad de México: Trillas, 1986

[6] Paz-Penagos, H., Aprendizaje autónomo y estilo cognitivo: Diseño didáctico, metodología y evaluación. Revista Educación en Ingenieía, 9, pp. 53-65. 2014.

[7] Romero, C. y Rosero, M., Modelo de enseñanza y su relación con los procesos metacognitivos en programación de sistemas. Revista Educación en Ingeniería, 9(17), pp. 1-12, 2014.

[8] Azad, A. and Smith, D., Teaching an introductory programming language in a general education course. 2014, Journal of Information Technology Education: Innovations in Practice, 13, pp. 57-67, 2014

[9] Small, G., El cerebro digital. Madrid. Editorial Urano, 2011.

[10] Costa, M. y Costa, F., Metodología da pesquisa: Preguntas e resposas. 1a Ed. Sao Paulo. DoAutor, 2013.

[11] Felleisen, M. et al., How to design programs. 2a Ed. Boston: MIT Press, 2013.

[12] Deitel and Deitel, C++ Programming. New York: Prentice Hall, 2013.

[13] Schildt, H., C Programming. México: McGraw Hill, 2010.

[14] Van Roy, P., Concepts, Techniques and models of computer programming. Estocolmo. Université Catholique de Louvain, 2008.

[15] Van Roy, P., Techniques and methods in programming computer. Louvaine: University Press, 2008.

[16] Paz-Penagos, H., ¿How to develop metacognition through problem solving in higher education? Revista de Ingeniería e Investigación, 31, pp. 75-80, 2009.

[17] Sparks, J., The pythatorean theorem. Bloomington (Indiana): AuthorHouse, 2008.

[18] Bruner, J.S., Hacia un teoría de la instrucción. Ciudad de México: Hispanoamericana, 1969.

[19] Trejos, O.I., Aprendizaje en ingeniería: Un problema de comunicación. Pereira (Colombia): Tesis Doctoral, Universidad Tecnológica de Pereira, Pereira, Colombia, 2012.

[20] Trejos, O.I., Fundamentos de programación. Pereira: Papiro, 2006. 
[21] Schildt, H., C++ programming. Vancouver: McGraw Hill, 2010.

[22] Trejos-Buriticá, O.I., Algoritmos problemas básicos. Pereira (Colombia): Papiro, 2008.

[23] Boyer, C., Historia de la matemática. Madrid (España): Alianza Editorial, 2010.

[24] Stewart, I., Historia de las matemáticas en los últimos 10000 años. Barcelona (España): Editorial Crítica, 2012.

[25] Attard, A. et al., Student centered learning. An insight into theory and practice. Bucarest: Lifelong learning programme - European Community, 2010.

[26] O'Farrel, C., Enhancing student learning through assesament. New York: Electronic Press, 2012.

[27] Vigotsky, L., El desarrollo de los procesos sicológicos superiores. México: Eitorial Grijalbo, 1981.

O.I. Trejos-Buriticá, es Ing. de Sistemas, Universidad Incca de Colombia (1992). Esp. en Instrumentación Física, Universidad Tecnológica de Pereira (2000). MSc. en Comunicación Educativa, Universidad Tecnológica de Pereira (2006). PhD en Ciencias de la Educación, Universidad Tecnológica de Pereira (2012). Docente de planta, Facultad de Ingenierías, Universidad Tecnológica de Pereira (1996). ExDirector de Ingeniería de Sistemas y Computación. ExDecano Facultad de Ingenierías, Universidad Tecnológica de Pereira. ORCID: 0000-0002-3751-6014 